\title{
Checklist of jumping plant-lice (Hemiptera, Sternorrhyncha, Psyllidae) from Mato Grosso, Brazil
}

\author{
Checklist de psilídeos (Hemiptera, Sternorrhyncha, Psylloidea) de Mato Grosso, \\ Brasil
}

\section{Marliton Rocha BarretoI, Dalva Luiz de Queiroz" Daniel Burckhardt ${ }^{\mathrm{III}}$, Luís Amilton Foerster ${ }^{\mathrm{IV}}$}

\begin{abstract}
Psyllids or jumping plant-lice are small phloem-feeding insects characterised by narrow host ranges, i.e., the plants on which they develop. This work shows the results of a survey of the superfamily Psylloidea in different biomes of Mato Grosso state. In total, 29 described genera and 24 described species are listed. The Cerrado biome showed the highest diversity, followed by the Amazon and Pantanal. Compared with a checklist published for Brazil in 2012, the present paper increases significantly the knowledge on the biodiversity of the state of Mato Grosso. In addition to the species recorded here for the first time from Mato Grosso (Diaphorina citri, Heteropsylla caldwelli, Pseudophacopteron longicaudatum, Tainarys myracrodrui and Trioza struthanthi), we provide also new records for other Brazilian states: Isogonoceraia divergipennis from Maranhão, Rio Grande do Sul, Rio de Janeiro and Santa Catarina; Heteropsylla caldwelli - from Goiás and São Paulo; Euphalerus clitoriae - from Ceará, Goiás, Pará, Paraná and Santa Catarina; Trioza tabebuiae - from Minas Gerais, São Paulo and Santa Catarina; and Trioza struthanthi from São Paulo.
\end{abstract}

Keywords: Biodiversity; Brazilian biomes; Distribution; Insecta

\section{Resumo}

Os psilídeos são insetos pequenos que se alimentam do floema e se caracterizam por apresentarem uma pequena faixa de plantas hospedeiras em que se desenvolvem. Este trabalho teve como objetivo prospectar e identificar insetos da superfamília de Psylloidea que ocorrem associados a diferentes biomas no Mato Grosso e que resultou na coleta de 29 gêneros descritos, com 24 espécies identificadas. O Cerrado foi o bioma que apresentou a maior diversidade, seguido pela Amazônia e Pantanal. Comparando com a última lista de psilídeos publicada para o Brasil, em 2012, este artigo contribuiu significativamente para o aumento do conhecimento da biodiversidade de Mato Grosso. Além das espécies registradas para o Mato Grosso (Diaphorina citri, Heteropsylla caldwelli, Pseudophacopteron longicaudatum, Tainarys myracrodruie Trioza struthanthi), também apresentamos novos registros para outros estados brasileiros: Isogonoceraia divergipennis- no Maranhão, Rio Grande do Sul, Rio de Janeiro e Santa Catarina; Heteropsylla caldwelli - em Goiás e São Paulo; Euphalerus clitoriae - no Ceará, Goiás, Pará, Paraná e Santa Catarina; Trioza tabebuiae em Minas Gerais, São Paulo e Santa Catarina; e Trioza struthanthi em São Paulo.

Palavras-chave: Biodiversidade; Biomas brasileiros; Distribuição; Insecta

Biólogo, Dr., Professor do Programa de Pós-Graduação em Ciências Ambientais, Universidade Federal de Mato Grosso, Av. Alexandre Ferronato, 1200, CEP 78550-728, Sinop (MT), Brasil. mrb.ufmt@gmail.com (ORCID: 0000-0003-3793-8855)

"I Engenheira Florestal, Dra., Pesquisadora da Empresa Brasileira de Pesquisa Agropecuária - Embrapa Florestas, Estrada da Ribeira, Km 111, CEP 83411-000, Colombo (PR), Brasil. dalva.queiroz@embrapa.br (ORCID: 0000-0003-3556-9910)

III Entomólogo, Dr., Curador em Naturhistorisches Museum, Augustinergasse 2, 4001 Basel, Switzerland. daniel.burckhardt@bs.ch (ORCID: 00000001-8368-5268)

IV Engenheiro Agrônomo, Dr., Professor do Programa de Pós-Graduação em Agronomia, Universidade Federal do Paraná, Rua dos Funcionários, 1.540, CEP 80035-050, Curitiba (PR), Brasil. foerster@ufpr.br (ORCID: 0000-0002-5235-0614) 


\section{Introduction}

Mato Grosso, the third largest state of Brazil, is located in the Midwest of the country, and contains parts of the Amazon, Cerrado and Pantanal biomes. Along with this extensive territorial area, encompassing three different biomes and an immense plant diversity, it is expected that the insect fauna, especially phytophages such as psyllids, is very rich. Psyllids constitute the superfamily Psylloidea in the Hemiptera: Sternorrhyncha. They are phloem feeders and typically monophagous or oligophagous. Some species are pests in agriculture, forestry or horticulture (Burckhardt 1994; Hollis 2004; Hodkinson 2009; Burckhardt et al. 2014) and can cause severe damage to host plants, such as Glycaspis brimblecombei Moore, 1964 to Eucalyptus spp. Others, like Diaphorina citri Kuwayama, 1908 and Bactericera cockerelli (Šulc, 1909) are even more dangerous because they transmit plant pathogens that cause serious diseases of their hosts.

Burckhardt and Queiroz (2012) recorded 73 psyllid species from Brazil and only four species from Mato Grosso state. The first species of Psylloidea cited for MT was Apsyllopsis mexicana (CRAWFORD, 1914), as Limbopsylla beeryi (CALDWELL, 1944) by Brown and Hodkinson (1988). Twent years later, Santana (2008) reported for the first time the occurrence of Blastopsylla occidentalis Taylor, 1985 in Mato Grosso, followed by Peres Filho et al. (2011), who reported the presence of Glycaspis brimblecombei (MOORE, 1964) in the region of Cuiabá and Primavera do Leste. Euphalerus clitoriae (BURCKHARDT; GUAJARÁ, 2000) was first recorded in the state by Burckhardt and Queiroz (2012).

Limataphalara lautereri Burckhardt and Queiroz 2013, was described from the material collected in Mato Grosso (Tabaporã and Sinop) (BURCKHARDT; QUEIROZ, 2013). Isogonoceraia divergipennis White and Hodkinson, 1980, was reported from Mato Grosso in 2016 and Trioza tabebuiae BURCKHARDT; SANTANA, 2001 in 2017 and both were collected near Sorriso (MAZZARDO et al., 2016; 2017). Burckhardt and Queiroz (2020) recorded for Mato Grosso Apsyllopsis mexicana, Colophorina bororo, Colophorina tupi, Jataiba basifistula, Mitrapsylla copaiferae, Mitrapsylla gloriae, Platycorypha cultrata and Platycorypha leptopeus.

Knowing the severe damage caused by some species of this group, such as gall formation, leaf deformation, disease vectors, in different countries and continents, with huge economic losses, it is extremely important to know the species present in this large area that comprises the state of Mato Grosso, not only in plantations, but also in native areas. Thus, this work aimed to prospect and identify insects of the Psylloidea superfamily that occur associated with different biomes in Mato Grosso.

\section{Material and methods}

Twenty-two collections were performed from September 2013 to March 2019. The periodicity of the collections varied according to the wet and dry seasons in the region as well as the opportunity for field work. An entomological net was used to collect the insects, by sweeping the edges of planted and natural forest present in the study area. The collection sites were georeferenced and all information about the locality, type of vegetation and host plants were recorded in a field book. The plant names were revised according to Flora do Brasil.

The psyllids collected in the net were sucked with an entomological aspirator, stored in tubes containing 70\% non-denaturated ethanol and properly labelled: date of collection, location, geographic coordinates and name of collector. The collections were made with the permits of IBAMA/SISBIO numbers 13362 and 11832 - Permanent license for the collection of zoological material and 41169 - Permits for activities with scientific purpose for the preservation areas: Pantanal Mato-grossense National Park and Chapada dos Guimarães, MT state.

All samples were taken to the Southern Amazon Biological Collection Entomology Laboratory - ABAM at the Federal University of Mato Grosso - UFMT, Sinop University Campus, where, with the aid of a stereoscopic microscope, they were sorted and the adults (male and 
female) and the immatures were counted.

Specimens were identified by DB using the psyllid collection of the NHMB and following publications with identification keys and descriptions: families, subfamilies and genera: Brown and Hodkinson (1988), Burckhardt (1994), Burckhardt et al. (1999) and Burckhardt and Queiroz (2020); Diaphorina Kuwayama, 1908: Mathur (1975); Isogonoceraia Tuthill, 1964: White and Hodkinson (1980); Euphalerus Schwarz, 1904: Burckhardt and Guajará (2000); Pseudophacopteron Enderlein, 1921: Malenovskýet et al. (2015); Tainarys Brèthes, 1920: Burckhardt and Queiroz (2017); Trioza Foerster, 1848: Santana and Burckhardt (2001), Burckhardt et al. (2017). Vouchers are deposited in the following collections: DZUP - Coleção Entomológica Padre Jesus Santiago Moure, Centro Politécnico, Universidade Federal do Paraná, Curitiba, Paraná, Brazil; MZSP - Museu de Zoologia da Universidade de São Paulo, São Paulo, São Paulo, Brazil; NHMB Naturhistorisches Museum, Basel, Switzerland.

In the text, the following Brazilian states are cited: Acre (AC), Alagoas (AL), Amazonas (AM), Bahia (BA), Ceará (CE), Espírito Santo (ES), Goiás (GO), Maranhão (MA), Mato Grosso (MT), Mato Grosso do Sul (MS), Minas Gerais (MG), Pará (PA), Paraíba (PB), Paraná (PR), Pernambuco (PE), Piauí (PI), Rio de Janeiro (RJ), Rio Grande do Sul (RS), Roraima (RR), Santa Catarina (SC), São Paulo (SP), Sergipe (SE), Tocantins (TO) as well as Distrito Federal (DF).

\section{Results and discussion}

The collection areas involved the three biomes present in Mato Grosso and covered 30 municipalities (Acorizal, Alto Garças, Barão de Melgaço, Cáceres, Chapada dos Guimarães, Claudia, Colíder, Cotriguaçu, Cuiabá, Diamantino, Guarantã do Norte, Itaúba, Itiquira, Juína, Lucas do Rio Verde, Nobres, Nova Guarita, Nova Mutum, Nova Santa Helena, Nova Ubiratã, Poconé, Porto dos Gaúchos, Rosário Oeste, Santa Carmem, Santo Antônio do Leverger, Sinop, Sorriso, Tabaporã, Terra Nova do Norte and Vera) resulting in the collection of 29 described genera (Allophorina*, Apsyllopsis, Blastopsylla, Calophya*, Caradocia ${ }^{*}$, Ciriacremum*, Colophorina, Diaphorina, Diclidophlebia, Epiacizzia*, Euceropsylla, Euphalerus, Euryconus, Glycaspis, Heteropsylla, Isogonoceraia, Jataiba, Leuronota, Limataphalara, Mastigimas, Mitrapsylla, Padaukia, Paracarsidara, Phacosemoides, Platycorypha, Pseudophacopteron, Trioza, Triozoida, and Tuthillia) ( ${ }^{*}$ genera without named species in $\mathrm{MT}$ ) and several undescribed genera.

Over 50 species were collected in the state of Mato Grosso, many which umdescribed. Of these, 24 described species were identified, five of them new to the state of Mato Grosso: Diaphorina citri Kuwayama, 1908, Heteropsylla caldwelli Burckhardt, 1987, Pseudophacopteron longicaudatum Malenovský et al., 2015, Tainarys myracrodrui Burckhardt and Queiroz, 2017 and Trioza struthanthi Burckhardt et al. 2017 (Figure 1). For these species, the distribution is revised, being detailed by states in Brazil and municipalities in MT state, adding, for some of them, new records for other Brazilian states, as presented below.

Aphalaridae

Aphalarinae

Limataphalara lautereri Burckhardt and Queiroz, 2013.

Distribution. Brazil: MS, MT (Sinop $11^{\circ} 52^{\prime} 17.2^{\prime \prime S}$, $55^{\circ} 35^{\prime} 44.6^{\prime \prime} \mathrm{W}$; Sorriso $12^{\circ} 25^{\prime} 43.5^{\prime \prime}$, $55^{\circ} 47^{\prime} 47.5^{\prime \prime W}$; $12^{\circ} 25^{\prime} 42.3^{\prime \prime S}, 55^{\circ} 47^{\prime} 46.8^{\prime \prime} \mathrm{W} ; 12^{\circ} 25^{\prime} 27.8^{\prime \prime S}, 55^{\circ} 51^{\prime} 01.2^{\prime \prime W}$; Tabaporã $11^{\circ} 18.828^{\prime} \mathrm{S}$, $55^{\circ} 57.691^{\prime} \mathrm{W} ; 11^{\circ} 20^{\prime} 00.0^{\prime \prime S}, 55^{\circ} 50^{\prime} 30.0^{\prime \prime W}$ ), PA (BURCKHARDT; QUEIROZ, 2013). Vouchers: NHMB 00002992, NHMB 00002993 , NHMB 00003024.

Host-plant. Nectandra cuspidata Nees and Mart. (Lauraceae) (BURCKHARDT; QUEIROZ, 2013).

Rhinocolinae

Tainarys myracrodrui Burckhardt and Queiroz, 2017.

New record. MT (Cuiabá $15^{\circ} 36^{\prime} 34.6^{\prime \prime S}$, 56 $03^{\circ} 54.7^{\prime \prime W}$; road MT 251 to Chapada dos Guimarães, 
Restaurante Chapadão, 170 m, 25.vii.2018, road side vegetation, Queiroz D. L.). Vouchers: NHMB. Distribution. Brazil: DF, MG, MS, SP (BURCKHARDT; QUEIROZ, 2017).

Host plant. Astronium graveolens Jacq., Myracrodruonu rundeuva Allemão (Anacardiaceae) (BURCKHARDT; QUEIROZ, 2013).

Spondyliaspidinae

Blastopsylla occidentalis Taylor, 1985.

Distribution. Originating from Australia, introduced into New Zealand and several countries in Africa, the Americas, Asia and Europe (OUVRARD, 2019). Brazil: BA, CE (QUEIROZ et al., 2018), GO (BURCKHARDT et al., 1999), MG, MS (BURCKHARDT; QUEIROZ, 2012), MT (Cuiabá $15^{\circ} 36^{\prime} 34.6^{\prime \prime S}, 56^{\circ} 03^{\prime} 54.7^{\prime \prime} \mathrm{W}$ - SANTANA, 2008), Poconé $16^{\circ} 21^{\prime} 09.7^{\prime \prime S}, 56^{\circ} 28^{\prime} 32.9^{\prime \prime} \mathrm{W}$; Sinop $11^{\circ} 51^{\prime} 45.5^{\prime \prime}$ S, $55^{\circ} 22^{\prime} 43.8^{\prime \prime W}$; Sorriso $12^{\circ} 23^{\prime} 35.34^{\prime \prime}$ S, $055^{\circ} 47^{\prime} 33.50^{\prime \prime W}$ ), PA (SALIBA et al., 2019) PR, SP (SANTANA, 2008), RS (QUEIROZ et al., 2018). Vouchers: NHMB.

Host-plant. Several species of Corymbia and Eucalyptus (Myrtaceae) (BURCKHARDT et al., 1999; OUVRARD, 2019).

Glycaspis brimblecombei Moore, 1964.

Distribution. Originating from Australia, introduced into New Zealand and several countries in Africa, the Americas, Asia and Europe (OUVRARD, 2019), Brazil: BA, DF, ES, GO, MG, MS, MT (Sinop $11^{\circ} 51^{\prime} 45.5^{\prime \prime} \mathrm{S}, 55^{\circ} 22^{\prime} 43.8^{\prime \prime} \mathrm{W}$; Sorriso $12^{\circ} 25^{\prime} 42.3^{\prime \prime} \mathrm{S}, 55^{\circ} 47^{\prime} 46.8^{\prime \prime} \mathrm{W}$ ), PA (SALIBA et al., 2019) PE, PR, RS, SC, SP, TO (BURCKHARDT; QUEIROZ, 2012; SILVA et al., 2013). Vouchers: NHMB 00003055.

Host-plant. Eucalyptus sp. (Myrtaceae).

Calophyidae

Mastigimatinae

Mastigimas anjosi Burckhardt et al. 2011.

Distribution. Trinidad, Venezuela (BURCKHARDT; QUEIROZ; DROHOJOWSKA, 2013), Brazil: MG, MT (Sinop 12 $23^{\prime} 35.34^{\prime \prime S}, 55^{\circ} 47^{\prime} 33.50^{\prime \prime W}$; Sorriso $12^{\circ} 25^{\prime} 38.10^{\prime \prime} \mathrm{S}, 55^{\circ} 47^{\prime} 44.60^{\prime \prime W}$ ), PR, RS, SP (BURCKHARDT et al., 2011; BURCKHARDT; QUEIROZ, 2012; BURCKHARDT; QUEIROZ; DROHOJOWSKA, 2013). Vouchers: NMB-PSYLL0005885.

Host-plant. Cedrela fissilis Vell. and Toona ciliata M. Roem. var. australis (Meliaceae) e (QUEIROZ; BURCKHARDT; ANJOS, 2013).

Liviidae

Euphyllurinae

Diaphorina citri Kuwayama, 1908.

New record. MT (Cuiabá $15^{\circ} 34^{\prime} 21.4^{\prime \prime}$ S, 56 $05^{\circ} 26.1^{\prime \prime W}$, 22.viii.2012, 180 m, D. L. Queiroz). Vouchers: NHMB DLQ333.

Distribution. Originating from Asia, introduced into the Americas, Iran, the Arabian Peninsula, Réunion, Kenya, Nigéria and Tanzania (OUVRARD, 2019). In Brazil: AL, BA, CE, GO, MA, MG, PA, PE, PR, RJ, RR, SE, SP (BURCKHARDT; QUEIROZ, 2012; FARIAS et al., 2018).

Host-plant. Citrus spp., Murraya paniculata (L.) (Rutaceae) (HALBERT; MANJUNATH, 2014).

Phacopteronidae

Phacosemoides sicki Costa Lima and Guitton, 1962.

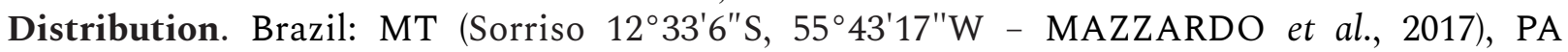
(BURCKHARDT; QUEIROZ, 2012). Vouchers: NHMB 00002999, NHMB 00003000, NHMB 00003001, NHMB 00003002.

Host-plant. Unknown (BURCKHARDT; QUEIROZ, 2012). 
Pseudophacopteron longicaudatum Malenovský et al. 2015.

New record. MT (Cuiabá - 15³4'36.0"S, 5606'06.0"W, 200 m, 3.xi.2012, D. L. Queiroz 57). Vouchers: NHMB \#DLQ358.

Distribution. Brazil: DF, GO, MG, MS, PR, SP (MALENOVSKÝ et al., 2015).

Host plant. Aspidosperma tomentosum Mart. and Zucc. and A. macrocarpon Mart. and Zucc. (Apocynaceae) (MALENOVSKÝ et al., 2015).

Psyllidae

Ciriacreminae

Isogonoceraia divergipennis White and Hodkinson, 1980.

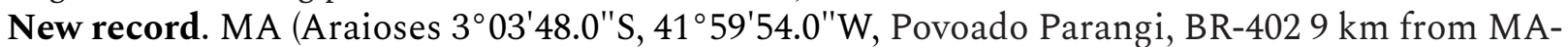
PI border, 30 m, 29.vi.2016); RS (Barra do Guaraí 30¹2'28.1"S, 57³3'31.0"W, Saladeira/along Rio Quará, Burckhardt D. and Queiroz D. L.); RJ (Itatiaia $22^{\circ} 27^{\prime} 45.4^{\prime \prime} S, 44^{\circ} 35^{\prime} 33.0^{\prime \prime} \mathrm{W}$, Parque Nacional do Itatiaia, park entrance, $600 \mathrm{~m}, 16-17 . i v .2019$, degraded Atlantic forest, Burckhardt D. and Queiroz D. L.; $22^{\circ} 28^{\prime} 57.7^{\prime \prime S}, 44^{\circ} 34^{\prime} 14.2^{\prime \prime} \mathrm{W}$, Parque Nacional do Itatiaia, park entrance, 16-17. iv.2019, degraded Atlantic forest, Burckhardt D. and Queiroz D. L.; $22^{\circ} 27^{\prime} 45.4^{\prime \prime S}, 44^{\circ} 35^{\prime} 33.0^{\prime \prime} \mathrm{W}$, Parque Nacional do Itatiaia, park entrance, 16-17.iv.2019, degraded Atlantic forest;) SC (Pomerode $26^{\circ} 44^{\prime} 42.0^{\prime \prime S}, 4^{\circ} 10^{\prime} 36.0^{\prime \prime} \mathrm{W}, 60-110 \mathrm{~m}, 29 . \mathrm{iv} .2013$, park vegetation, degraded Atlantic forest; sweeping vegetation, Burckhardt D. and Queiroz D. L.). Vouchers: NMB-PSYLL0005240, NMB-PSYLL0005226.

Distribution. Brazil: BA (WHITE; HODKINSON 1980), MG, MT (Sorriso $12^{\circ} 23^{\prime} 35.34^{\prime \prime}$, $55^{\circ} 47^{\prime} 33.50^{\prime \prime} \mathrm{W}$ - MAZZARDO et al. 2016: 2017), Cáceres $16^{\circ} 08^{\prime} 02.5^{\prime \prime S}, 57^{\circ} 41^{\prime} 34.4^{\prime \prime} \mathrm{W}$; $16^{\circ} 07^{\prime} 48.0^{\prime \prime S}, 57^{\circ} 41^{\prime} 02.6^{\prime \prime} \mathrm{W}$; Chapada dos Guimarães $15^{\circ} 27^{\prime} 54.60^{\prime \prime} \mathrm{S}, 55^{\circ} 43^{\prime} 11.90^{\prime \prime W}$; Poconé $15^{\circ} 56^{\prime} 11.7^{\prime \prime S}, 56^{\circ} 55^{\prime} 55.8^{\prime \prime W}$; Sinop $11^{\circ} 51^{\prime} 06.2^{\prime \prime S}, 55^{\circ} 31^{\prime} 31.9^{\prime \prime W}$; $\left.12^{\circ} 00^{\prime} 62,4^{\prime \prime S}, 55^{\circ} 14^{\prime} 35,4^{\prime \prime W}\right)$, PR, SP (SANTANA; BURCKHARDT, 2002; BURCKHARDT; QUEIROZ, 2012).

Host-plant. Cenostigma pluviosum (DC.) E. Gagnon and G.P. Lewis var. pluviosum (Fabaceae) (BURCKHARDT; QUEIROZ, 2012).

Heteropsylla caldwelli Burckhardt, 1987.

New record. GO (Alto Paraíso do Goiás $14^{\circ} 09^{\prime} 37.4^{\prime \prime S}$, $47^{\circ} 47^{\prime} 29.4^{\prime \prime} \mathrm{W}$, near São Jorge, Parque Nacional da Chapada dos Veadeiros, around researchers' accommodations, $1060 \mathrm{~m}$, 15.ii.2018, cerrado vegetation, planted trees, Queiroz D. L. and Burckhardt D.), MT (Cáceres $16^{\circ} 02^{\prime} 23.7^{\prime \prime S}$, $57^{\circ} 33^{\prime} 57.8^{\prime \prime W}, 144 \mathrm{~m}, 9.1 i i .2017$, Ben-Hur, R. e Barreto, M.R. \#455); SP (Piracicaba $22^{\circ} 42^{\prime} 38.9^{\prime \prime}$, $47^{\circ} 37^{\prime} 55.9^{\prime \prime W}$, Universidade de São Paulo, ESALQ (Escola Superior de Agricultura "Luiz de Queiroz") campus, $550 \mathrm{~m}$, 6.ii.2018, park vegetation; Santa Maria da Serra $22^{\circ} 40^{\prime} 56.3^{\prime \prime S}$, $48^{\circ} 18^{\prime} 17.6^{\prime \prime W}$, Mina Velha, $450 \mathrm{~m}, 7.1 i .2018$, vegetable and fruit gardens, edge of cerrado; Queiroz D. L. and Burckhardt D.). Vouchers: NMB-PSYLL0005236.

Distribution. Brazil: MG, PR, RS (MACHADO et al., 2017).

Host-plant. Albizia edwallii (Hoehne) Barneby and J. W. Grimes, Albizia hassleri (Chodat), Anadenanthera colubrina (Vell.) Brenan, Anadenanthera peregrina var. falcata (Benth.), Enterolobium contortisiliquum (Vell.), Senegalia polyphylla (DC.) Britton and Rose (Fabaceae) (MACHADO et al., 2017).

Jataiba basifistula Burckhardt and Queiroz 2020.

Distribution. Brazil: AC, MT (Rosário Oeste 14³1'41.5"S, 5548'16.9"W) (BURCKHARDT; QUEIROZ, 2020). Vouchers: NHMB.

Host plant. Unknown (BURCKHARDT; QUEIROZ, 2020).

Mitrapsylla copaiferae Burckhardt and Queiroz 2020.

Distribution. Brazil: GO, MG, MT (Barão de Melgaço 16²0'18.6"S, 5549'18.1"W; Nova Mutum $13^{\circ} 47^{\prime} 16.1^{\prime \prime S}$, $56^{\circ} 17^{\prime} 25.8^{\prime \prime} \mathrm{W}$; Sorriso $12^{\circ} 32^{\prime} 46.0^{\prime \prime S}, 55^{\circ} 43^{\prime} 32.2^{\prime \prime W} ; 12^{\circ} 25^{\prime} 30.0^{\prime \prime S}, 55^{\circ} 47^{\prime} 48.1^{\prime \prime} \mathrm{W}$; 
$12^{\circ} 24^{\prime} 19.1^{\prime \prime S}, 55^{\circ} 49^{\prime} 25.7^{\prime \prime} \mathrm{W} ; 12^{\circ} 24^{\prime} 04.0^{\prime \prime} \mathrm{S}, 55^{\circ} 49^{\prime} 24.2^{\prime \prime} \mathrm{W}$ ), MS, SP (BURCKHARDT; QUEIROZ, 2020). Vouchers: MZSP \#141(4)

Host-plant. Copaifera langsdorffii Desf., perhaps also Copaifera marginata Benth (BURCKHARDT; QUEIROZ, 2020).

Mitrapsylla gloriae Burckhardt and Queiroz 2020.

Distribution. Brazil: DF, GO, MT (Barão de Melgaço $16^{\circ} 20^{\prime} 18.6^{\prime \prime} \mathrm{S}, 55^{\circ} 49^{\prime} 18.1^{\prime \prime} \mathrm{W}$; Cuiabá $15^{\circ} 20^{\prime} 43.1^{\prime \prime S}, 55^{\circ} 50^{\prime} 42.0^{\prime \prime} \mathrm{W}$; Itiquira $17^{\circ} 30^{\prime} 50.0^{\prime \prime S}$, $\left.54^{\circ} 44^{\prime} 24.4^{\prime \prime} \mathrm{W}\right)$, MS, MG, PR, SC, SP (BURCKHARDT; QUEIROZ, 2020). Vouchers: NMB-PSYLL0004730, NMB-PSYLL0004854.

Host-plant. Copaifera langsdorffi Desf., C. marginata Benth. (Fabaceae) (BURCKHARDT; QUEIROZ, 2020).

\section{Macrocorsinae}

Apsyllopsis mexicana (Crawford 1914).

Distribution. Mexico and Panama (BURCKHARDT; QUEIROZ, 2020). Brazil: CE, GO, MA,

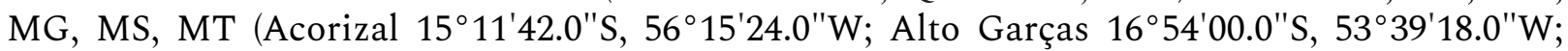
Chapada dos Guimarães $15^{\circ} 28^{\prime} 19.30^{\prime \prime S}, 55^{\circ} 45^{\prime} 57.70^{\prime \prime W}$; $15^{\circ} 28^{\prime} 05.10^{\prime \prime S}, 55^{\circ} 45^{\prime} 32.00^{\prime \prime W}$; Cuiabá $15^{\circ} 36^{\prime} 34.6^{\prime \prime S}, 56^{\circ} 03^{\prime} 54.7^{\prime \prime W}$; Diamantino $13^{\circ} 50^{\prime} 58.70^{\prime \prime S}, 56^{\circ} 28^{\prime} 51.50^{\prime W}$; Guarantã do Norte $09^{\circ} 57^{\prime} 44.0^{\prime \prime S}, 54^{\circ} 53^{\prime} 24.8^{\prime \prime W}$; Lucas do Rio Verde $13^{\circ} 07^{\prime} 01.60^{\prime \prime S}, 55^{\circ} 53^{\prime} 52.10^{\prime \prime W}$; Nobres $14^{\circ} 34^{\prime} 13.60^{\prime \prime S}$, $55^{\circ} 51^{\prime} 23.30^{\prime \prime} \mathrm{W}$; Nova Guarita $10^{\circ} 13^{\prime} 16.90^{\prime \prime S}, 55^{\circ} 22^{\prime} 52.40^{\prime \prime} \mathrm{W} ; 10^{\circ} 12^{\prime} 04.70^{\prime \prime S}$, $55^{\circ} 25^{\prime} 52.20^{\prime \prime} \mathrm{W}$; Nova Mutum $13^{\circ} 42^{\prime} 08.30^{\prime \prime S}, 56^{\circ} 04^{\prime} 16.30^{\prime \prime} \mathrm{W} ; 13^{\circ} 47^{\prime} 50.60^{\prime \prime S}, 56^{\circ} 01^{\prime} 41.10^{\prime \prime W}$; $13^{\circ} 47^{\prime} 35.80^{\prime \prime S}, 55^{\circ} 56^{\prime} 09.20^{\prime \prime} \mathrm{W}$; Poconé, Santo Antônio do Leverger $16^{\circ} 14^{\prime} 43.8^{\prime \prime S}, 55^{\circ} 47^{\prime} 05.3^{\prime \prime W}$; Sinop $11^{\circ} 51^{\prime} 54.0^{\prime \prime S}, 55^{\circ} 31^{\prime} 18.0^{\prime \prime} \mathrm{W}$; Sorriso $12^{\circ} 25^{\prime} 34.00^{\prime \prime S}, 55^{\circ} 47^{\prime} 39.70^{\prime \prime W}$; Terra Nova do Norte $10^{\circ} 36^{\prime} 23.10^{\prime \prime S}, 55^{\circ} 04^{\prime} 17.90^{\prime \prime W}$ ), PA (BURCKHARDT; QUEIROZ, 2012; 2020). Vouchers: NHMB 00003041, NMB-PSYLL0005709, NMB-PSYLL0005734, NMB-PSYLL0005751.

Host-plant. Hymenaea spp. (Fabaceae) (BURCKHARDT; QUEIROZ, 2020).

Colophorina bororo Burckhardt and Queiroz 2020.

Distribution.Brazil: MT (Sorriso $12^{\circ} 24^{\prime} 03.2^{\prime \prime S}, 55^{\circ} 49^{\prime} 23.9^{\prime \prime} \mathrm{W} ; 12^{\circ} 24^{\prime} 19.8^{\prime \prime} \mathrm{S}, 55^{\circ} 49^{\prime} 25.7^{\prime \prime} \mathrm{W}$ (BURCKHARDT; QUEIROZ, 2020). Vouchers: NMB-PSYLL0006498, NMB-PSYLL0006499.

Host-plant. Copaifera oblongifolia Mart. (Fabaceae) (BURCKHARDT; QUEIROZ, 2020).

Colophorina tupi Burckhardt and Queiroz 2020.

Distribution. Brazil: BA, GO, MG, MS, MT (Barão de Melgaço 16²0'18.6"S, 55²9'18.1"W; Cuiabá $15^{\circ} 20^{\prime} 43.1^{\prime \prime} S, 55^{\circ} 50^{\prime} 42.0^{\prime \prime} \mathrm{W}$ ), PR, SP (BURCKHARDT; QUEIROZ, 2020). Vouchers: NMB-PSYLL0005634, NHMB 00003039.

Host-plant. Copaifera langsdorffii Desf. (Fabaceae) (BURCKHARDT; QUEIROZ, 2020).

Euphalerus clitoriae Burckhardt and Guajará 2000.

New record. CE (Tianguá $3^{\circ} 39^{\prime} 36.0^{\prime \prime S}, 40^{\circ} 56^{\prime} 54.0^{\prime \prime} \mathrm{W}$; Serra da Ibiapaba, BR-222 km 302, $480 \mathrm{~m}$, 4.vii.2016; sweeping vegetation, Queiroz D. L. and Burckhardt D.), GO (Mossâmedes, $16^{\circ} 07^{\prime} 04.8^{\prime \prime S}$, $50^{\circ} 12^{\prime} 14.4^{\prime \prime} \mathrm{W}, 690 \mathrm{~m}, 20.1 i .2018$, isolated trees in city and along pasture; sweeping vegetation), PA (Belém 1 ${ }^{\circ} 24^{\prime} 54.0^{\prime \prime S}, 48^{\circ} 25^{\prime} 18.0^{\prime \prime} \mathrm{W}$, Embrapa campus, $20 \mathrm{~m}, 8-15 . i v .2013$, plantations and edge of degraded Amazonian forest; sweeping vegetation, Queiroz D. L. and Burckhardt D.), PR (Antonina $25^{\circ} 14^{\prime} 37.8^{\prime \prime S}, 48^{\circ} 45^{\prime} 04.0^{\prime \prime} \mathrm{W}$, Usina Parigot de Souza, $28 \mathrm{~m}, 17-20 . v i i .2017$, roadside vegetation, Atlantic forest; sweeping vegetation, Queiroz D. L. and Burckhardt D.), SC (Pomerode $26^{\circ} 46^{\prime} 30.0^{\prime \prime} \mathrm{S}, 49^{\circ} 13^{\prime} 06.0^{\prime \prime} \mathrm{W}, 60-110 \mathrm{~m}, 29 . \mathrm{iv} .2013$, park vegetation, degraded Atlantic forest; sweeping vegetation, Queiroz D. L. and Burckhardt D.). Vouchers: NHMB 00003040, NMBPSYLL0005718, NMB-PSYLL0005715, NMB-PSYLL0005748, NMB-PSYLL0005213.

Distribution. Brazil: AL, BA, MG, MT (Cuiabá $15^{\circ} 34^{\prime} 21.4^{\prime \prime S}, 56^{\circ} 05^{\prime} 26.1^{\prime \prime W}$; Nobres $14^{\circ} 32^{\prime} 41.70^{\prime \prime S}$, $55^{\circ} 51^{\prime} 46.60^{\prime \prime} \mathrm{W} ; 14^{\circ} 32^{\prime} 43.30^{\prime \prime} \mathrm{S}, 55^{\circ} 51^{\prime} 50.40^{\prime \prime} \mathrm{W}$; $14^{\circ} 34^{\prime} 13.60^{\prime \prime} \mathrm{S}, 55^{\circ} 51^{\prime} 23.30^{\prime \prime W}$; Rosário Oeste 
$14^{\circ} 34^{\prime} 13.6^{\prime \prime S}, 55^{\circ} 51^{\prime} 23.3^{\prime \prime W}$; Sinop, Sorriso $12^{\circ} 25^{\prime} 31.1^{\prime \prime S}, 55^{\circ} 51^{\prime} 01.8^{\prime \prime W}$; Tabaporã $11^{\circ} 18^{\prime} 48.0^{\prime \prime S}$, $55^{\circ} 57^{\prime} 42.0^{\prime \prime W}$ ), SP, PE, RJ (BURCKHARDT; QUEIROZ, 2012).

Host-plant. Clitoria fairchildiana R. A. Howard (Fabaceae) (BURCKHARDT; QUEIROZ, 2012).

Psyllinae

Padaukia macrolobii Burckhardt and Queiroz 2018.

Distribution. AM, MT (Colider 10³2'23.60"S, 55 ${ }^{\circ} 28^{\prime} 38.20^{\prime \prime W}$ ), RR (BURCKHARDT; QUEIROZ, 2018). Vouchers: MZSP \#128(5).

Host-plant. Macrolobium acaciifolium (Benth.) Benth. (Fabaceae, Caesalpinoideae) (BURCKHARDT; QUEIROZ, 2018).

Platycorypha cultrata Burckhardt and Queiroz 2020.

Distribution. Brazil: MT (Chapada dos Guimarães $15^{\circ} 28^{\prime} 19.2^{\prime \prime S}, 55^{\circ} 45^{\prime} 57.6^{\prime \prime W}$; $15^{\circ} 27^{\prime} 54.7^{\prime \prime S}$, $55^{\circ} 43^{\prime} 12.0^{\prime \prime} \mathrm{W}$; Cuiabá $15^{\circ} 36^{\prime} 34.6^{\prime \prime} \mathrm{S}, 56^{\circ} 03^{\prime} 54.7^{\prime \prime W}$; Nova Mutum $\left.13^{\circ} 35^{\prime} 34.8^{\prime \prime S}, 56^{\circ} 03^{\prime} 40.3^{\prime \prime W}\right)$ (BURCKHARDT; QUEIROZ, 2020). Vouchers: MZSP \#587.

Host plant. Adults were collected on Hymenaea courbaril L. (Fabaceae) which is a likely host (BURCKHARDT; QUEIROZ, 2020).

Platycorypha leptopeus Burckhardt and Queiroz 2020.

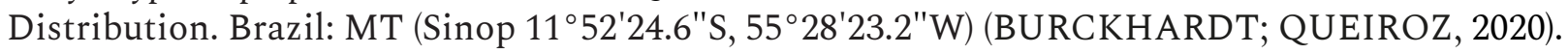
Vouchers: NMB-PSYLL0006443.

Host plant. Unknown (BURCKHARDT; QUEIROZ, 2020).

Platycorypha rostrata Burckhardt and Queiroz 2020.

Distribution. Brazil: MG, MS, MT (Nova Guarita $10^{\circ} 12^{\prime} 04.7^{\prime \prime S}, 55^{\circ} 25^{\prime} 52.3^{\prime \prime W}$; Sinop $11^{\circ} 51^{\prime} 06.5^{\prime \prime S}$, $55^{\circ} 30^{\prime} 03.6 " \mathrm{~W}$; Sorriso $12^{\circ} 32^{\prime} 46.0^{\prime \prime} \mathrm{S}$, 5543'32.2"W); PI (BURCKHARDT; QUEIROZ, 2019). Vouchers: NMB-PSY LL0006455, NMB-PSYLL0006456.

Host plant. Hymenaea (Fabaceae) which is a likely host (BURCKHARDT; QUEIROZ, 2020).

Triozidae

Trioza tabebuiae Burckhardt and Santana 2001.

New record. MG (Três Marias $18^{\circ} 13^{\prime} 42.0^{\prime \prime S}$, 4513'12.0"W, BR 040, km 250, 760 m, 10.vii.2012, scrub along road; sweeping vegetation, Queiroz D. L. and Burckhardt D.; Presidente Olegário $18^{\circ} 20^{\prime} 28.3^{\prime \prime S}, 46^{\circ} 29^{\prime} 47.0^{\prime \prime} \mathrm{W}$, between Presidente Olegário and Lagamar, $950 \mathrm{~m}$, 12.vii.2012, scrub along road; Queiroz D. L. and Burckhardt D.; Uberlândia, $19^{\circ} 11^{\prime} 01.3^{\prime \prime S}, 48^{\circ} 23^{\prime} 46.0^{\prime \prime} \mathrm{W}$, Panga, 810 m, 8.ii.2018, cerrado; Queiroz D. L. and Burckhardt D.), SP (Piracicaba $22^{\circ} 42^{\prime} 38.9^{\prime \prime S}$, $47^{\circ} 37^{\prime} 55.9^{\prime \prime} \mathrm{W}$; Queiroz D. L. and Burckhardt D.), SC (Pomerode $26^{\circ} 44^{\prime} 42.0^{\prime \prime} \mathrm{S}, 49^{\circ} 10^{\prime} 36.0^{\prime \prime} \mathrm{W}$, road Pomerode to Timbó, SC-416, 4 km from Timbó, Pousada Paraíso da Pesca, 270-380 m, 29.iv.2013, remnants of Atlantic forest; Queiroz D. L. and Burckhardt D.). Vouchers: NMB-PSYLL0005629, NMB-PSYLL0005625, NMB-PSYLL0005578, NMB-PSYLL0005643.

Distribution. Brazil: PA (BURCKHARDT; QUEIROZ, 2012), MT (Sorriso $12^{\circ} 24^{\prime} 08.10 " S$;

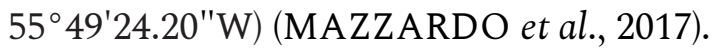

Host-plant. Handroanthus spp. (Bignoniaceae) (BURCKHARDT; QUEIROZ, 2012).

Trioza struthanthi Burckhardt et al. 2017.

New record: MT (Cuiabá $15^{\circ} 22^{\prime} 46.2^{\prime \prime}$ S, 55 $50^{\circ} 33.4^{\prime \prime W}$, road to PN Chapada dos Guimarães, Rio Paciência, 300 m, 17.iii.2019, Queiroz D. L.), SP (São José do Barreiro $22^{\circ} 44^{\prime} 49.9^{\prime \prime S}, 44^{\circ} 37^{\prime} 00.1^{\prime \prime} \mathrm{W}$, Queiroz D. L. and Burckhardt D.). Vouchers: NMB-PSYLL0004455, NMB-PSYLL0004456, NMBPSYLL0004457.

Distribution. Brazil: MG, PR, RS, SC (BURCKHARDT et al. 2017).

Host plant. Struthanthus uraguensis G. Don (Loranthaceae) (BURCKHARDT et al., 2017). 
Triozoida limbata (Enderlein, 1918).

Distribution. América Central and do Sul (OUVRARD, 2019). Brazil: AM, BA, CE, ES, MA, MG, MS, MT (Chapada dos Guimarães $15^{\circ} 27^{\prime} 54.60^{\prime \prime S}, 55^{\circ} 43^{\prime} 11.90^{\prime \prime W}$; Cotriguaçu 09 ${ }^{\circ} 51^{\prime} 29,1^{\prime \prime S}$, $058^{\circ} 14^{\prime} 92,7^{\prime \prime} \mathrm{W} ; \quad 09^{\circ} 51^{\prime} 17.50^{\prime \prime S}, \quad 58^{\circ} 14^{\prime} 55.60^{\prime \prime W} ; \quad 09^{\circ} 52.121^{\prime} \mathrm{S}, \quad 058^{\circ} 13.779^{\prime} \mathrm{W} ; \quad 09^{\circ} 52^{\prime} 10.1^{\prime \prime} \mathrm{S}$, $058^{\circ} 13^{\prime} 40.9^{\prime \prime} \mathrm{W} ; 09^{\circ} 52^{\prime} 10.70^{\prime \prime S}, 58^{\circ} 13^{\prime} 43.70^{\prime \prime W}$; $09^{\circ} 52^{\prime} 13.50^{\prime \prime S}, 58^{\circ} 13^{\prime} 42.90^{\prime \prime W}$; Cuiabá $15^{\circ} 34^{\prime} 21.4^{\prime \prime} \mathrm{S}$,

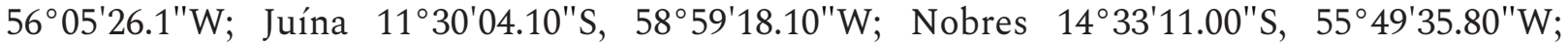
$14^{\circ} 33^{\prime} 00.90^{\prime \prime S}, 55^{\circ} 50^{\prime} 11.90^{\prime \prime W}$; Poconé $16^{\circ} 30^{\prime} 24.60^{\prime \prime S}$, 56 $\left.24^{\circ} 48.40^{\prime \prime W}\right)$, PA, PB, PE, PR, RJ, RR, SC, SP (QUEIROZ et al., 2018; OUVRARD, 2019). Vouchers: NMB-PSYLL0005560, NMBPSYLL0005635, NMB-PSYLL0005718.

Host-plant. Psidium guajava L. (Myrtaceae) (BURCKHARDT; QUEIROZ, 2012).

Figure 1 - Distribution of psyllid species in the state of Mato Grosso

Figura 1 - Distribuição de espécies de psilídeos no estado de Mato Grosso

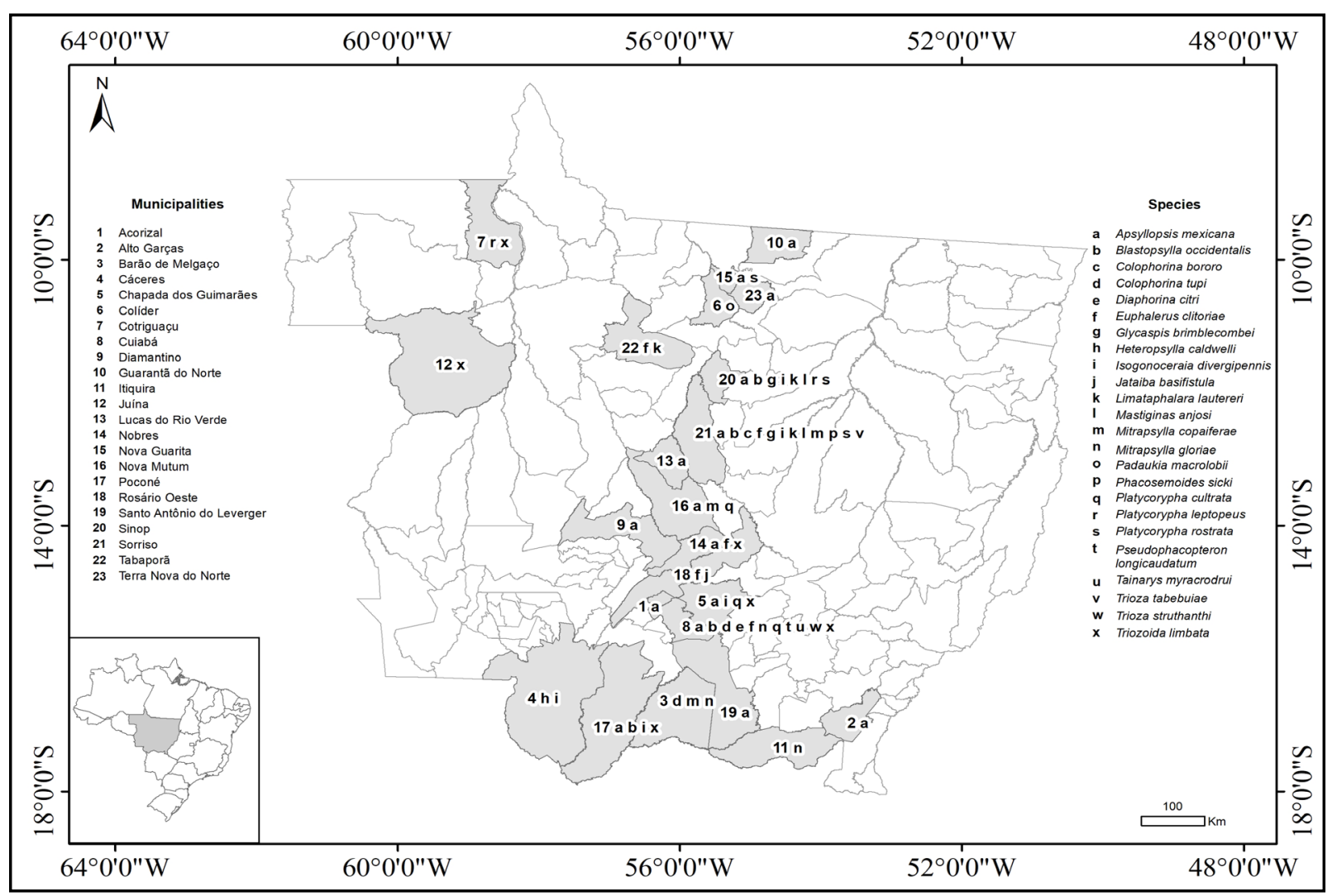

Source: Authors (2019)

Regarding the distribution of species in relation to biomes, four of the collected psyllids were found in the 3 regions. When analysed by biome, the Cerrado was the one with the highest diversity with 24 species collected, 09 of them only present in this biome, followed by the Amazon and the Pantanal with 18 and 8 species respectively. However, this may be an artefact of uneven collecting efforts per biome. The Cerrado biome was with $48 \%$ the most sampled, followed by the Amazon (32\%) and Pantanal (20\%).

Considering the Cerrado-forest transition region, in this area were collected over $70 \%$ of all species of this work. For Silva and Bates (2002), in the savannah, a mosaic of savannaforest transition regions corresponds to approximately $24 \%$ and dry forests correspond to $4 \%$ of the area. The regions comprised between the municipalities of Claudia and Gaúcha do Norte 
(MT), are in the so-called transition zone of the Amazon and savannah domains, forming the division of the vegetation biomas of MT, being an area of strong pressure from selective logging (NUNES; SILVA; FERRAZ, 2017), highlighting the need for biodiversity inventories. Mainly or secondarily, the Amazon biome is present in 93 municipalities, the savannah Biome in 92 and the Pantanal in 15 municipalities out of 141 of the state.

In the Pantanal, the dry season varies in intensity (LOPES et al., 2017) and depends on the distribution and amount of rainfall, which varies within and between years and sub-regions and is subject to burning, which brings additional stress to animals that inhabit the region, increasing its mortality. Although collections in the Pantanal were carried out in both dry and rainy seasons, few species (8) were found. Most psyllids are associated with new leaves and shoots with tender tissues. However, frequent heavy rains make establishment of insects on plants more difficult. In Pantanal the two seasons are well defined with one very dry and the other with torrential rain, it may be that the collections were not done at the best time to find the insect peaks. These results highlight the need for more collections in this biome, also observing the different phenological phases of the plants.

Species distribution is usually associated with the distribution of their host plants and, in the present study this was true for Apsyllopsis mexicana, Blastopsylla occidentalis, Isogonoceraia divergipennis and Triozoida limbata that were collected from their respective hosts in all biomes. However, it is important to emphasize that more collections need to be performed to better characterize the distribution of species as well as other species may be collected.

The information presented here also broadens the knowledge on the geographical distribution of some pest psyllids, such as Blastopsylla occidentalis, Diaphorina citri, Glycaspis brimblecombei, Mastigimas anjosi and Triozoida limbata, which are important for defining their coverage regions as well as for setting up the phytosanitary history based on pest fluctuation.

\section{Conclusion}

This article contributed 5 new records for the state of Mato Grosso. It also brings new records for the states of Ceará, Goiás, Maranhão, Minas Gerais, Pará, Paraná, Rio de Janeiro, Rio Grande do Sul, Santa Catarina and São Paulo.

\section{Acknowledgements}

For the support / funding: Conselho Nacional de Desenvolvimento Científico e Tecnológico Process 164887 / 2017-2, Pró-Reitoria de Pesquisa Project 417/2015. Fundação de Amparo à Pesquisa do Estado de Mato Grosso - Universal Notice 224315/2015. To the taxonomists M. L. Brotto and J. T. W. Motta (Municipal Botanical Museum, Curitiba, PR) for the identification of the host plants. For help in collecting: R. Ben-Hur (Universidade Federal de Mato Grosso - UFMT, Sinop, MT), T. Mazzardo (UFMT, Sinop, MT), L.A. Pezzini (UFMT, Sinop, MT), G.D. Cort (Embrapa, Colombo, PR), P.S.B. Pucci (Embrapa, Colombo, PR) and D.I. RendónMera (Universidade Federal do Paraná - UFPR, Curitiba, PR).The authors thank the anonymous reviewers.

\section{References}

BROWN, R. G.; HODKINSON, I. D. Taxonomy and ecology of the jumping plant-lice of Panama (Homoptera: Psylloidea). In: LYNEBORG, L. (ed). Entomonograph. Leiden; New York; Kopenhagen; Köln: Scandinavian Science Press, 1988. p. 304.

BURCKHARDT, D. Generic key to Chilean jumping plant-lice (Homoptera: Psylloidea) with inclusion of potential exotic pests. Revista Chilena de Entomología, Santiago, v. 21, p. 57-67, 
1994.

BURCKHARDT, D. Jumping plant lice (Homoptera: Psyllidae) of the temperate neotropical region. part 1: Psyllidae (subfamilies Aphalarinae, Rhinocolinae and Aphalaroidinae). Zoological Journal of the Linnean Society, London, v. 89, p. 299-392, 1987.

BURCKHARDT, D. et al. Four new neotropical Trioza species associated with Loranthaceae (Santalales) and comments on mistletoe inhabiting psyllids (Hemiptera, Psylloidea). Alpine Entomology, Sofia, v. 1, p. 91-108, 2017.

BURCKHARDT, D. et al. Psyllid host-plants (Hemiptera: Psylloidea): resolving a semantic problem. Florida Entomologist, 97, 242-246, 2014.

BURCKHARDT, D. et al. The jumping plant-louse Mastigimas anjosi spec. nov., a new pest of Toona ciliata (Meliaceae) in Brazil (Hemiptera, Psylloidea). Spixiana, München, v. 34, p. 109-120, 2011. Available from: https://www.academia.edu/35182010/The_jumping_plant-louse_Mastigimas_ anjosi_spec._nov._a_new_pest_of_Toona_ciliata_Meliaceae_in_Brazil. Accessed: 19 jul. 2020.

BURCKHARDT, D.; GUAJARÁ, M. Euphalerus clitoriae sp. n., a new psyllid species from Clitoria fairchildiana (Fabaceae, Papilionoideae), and notes on other Euphalerus spp. (Hemiptera, Psylloidea). Revue Suisse de Zoologie, Genève, v. 107, p. 325-334, 2000. DOI: 10.5962/bhl.part.80132

BURCKHARDT, D.; QUEIROZ, D. L. Checklist and comments on the jumping plant-lice (Hemiptera: Psylloidea) from Brazil. Zootaxa, Auckland, v. 3571, p. 26-48, 2012. Available from: http://zoobank.org/References/731C7CF3-8E5B-48DE-8297-E2853855825C. Accessed: 19 jul. 2020.

BURCKHARDT, D.; QUEIROZ, D. L. First record of the Old World genus Padaukia Hollis and Martin, 1993 from Brazil with description of a new species (Hemiptera: Psylloidea). Entomologische Zeitschrift, Schwanfeld, v. 128, p. 131-135, 2018.

BURCKHARDT, D.; QUEIROZ, D. L. The jumping plant-lice of the Neotropical genus Tainarys (Hemiptera: Psylloidea) associated with Anacardiaceae. Zootaxa, Auckland, v. 4232, p. 535-567, 2017. DOI: 10.11646/zootaxa.4232.4.5

BURCKHARDT, D.; QUEIROZ, D. L. Neotropical jumping plant-lice (Hemiptera, Psylloidea) associated with plants of the tribe Detarieae (Leguminosae, Detarioideae). Zootaxa, Auckland, v. 4733, p. 1-71, 2020.

BURCKHARDT, D.; QUEIROZ, D. L. Phylogenetic relationships within the subfamily A phalarinae including a revision of Limataphalara (Hemiptera: Psylloidea: Aphalaridae). Acta Musei Moraviae, Scientiae Biologicae, Brno Czech Republic, v. 98, p. 35-56, 2013. Available from: http:// www.mzm.cz/fileadmin/user_upload/publikace/casopisy/AMM_SB_98_2_2013/05Burckhardt_ Queiroz.pdf. Accessed: 01 mar. 2020.

BURCKHARDT, D.; QUEIROZ, D. L.; DROHOJOWSKA, J. Revision of the neotropical jumping plant-louse genus Mastigimas (Hemiptera, Psylloidea) attacking Cedrela and Toona species (Meliaceae). Zootaxa, Auckland, v. 3745, p. 001-018, 2013. DOI: http://dx.doi.org/10.11646/ zootaxa.3745.1.1

BURCKHARDT, D. et al. Psyllid pests (Hemiptera, Psylloidea) in South American eucalypt plantations. Mitteilungen der Schweizerischen Entomologischen Gesellschaft, Switzerland, v. 72, p. 1-10, 1999. DOI: http://doi.org/10.5169/seals-402733

COstA LiMA, A. M.; GUITTON, N. Novo inseto galicola, Phacosemoides sicki, g.n., sp.n. (Homoptera, Psyllidae, Ciriacreminae). Memórias do Instituto Oswaldo Cruz, Rio de Janeiro, v. 60, p. 219-224, 1962.

CRAWFORD, D. L. A monograph of the jumping plant-lice or Psyllidae of the new world. Washington: Government Printing Office, 1914. 186 p. 
ENDERLEIN, G. Psyllidologica V. Zoologische Jahrbücher. Abteilungfür Systematik. Geographie und Biologie der Tiere, Jena, v. 41, p. 479-486, 1918.

FARIAS, A. P. et al. Dinâmica populacional e parasitismo natural de Diaphorina citri Kuwayama (Hemiptera: Liviidae) em pomares de citros em Sergipe. EntomoBrasilis, Vassouras, v. 11, p. 2025, 2018. DOI: 10.12741/ebrasilis. v11i1. 720.

HALBERT, S. E.; MANJUNATH, K. L. Asian citrus psyllids (Sternorrhyncha: Psyllidae) and greening disease of citrus: a literature review and assessment of risk in Florida. Florida Entomologist, v. 87, p. 330-353, 2004.

HODKINSON, I. D. Life cycle variation and adaptation in jumping plant lice (Insecta: Hemiptera: Psylloidea): a global synthesis. Journal of Natural History, v. 43, p. 65-179, 2009.

HOLLIS, D. Australian Psylloidea: Jumping plantlice and lerp insects. Department of the Environment and Heritage, Canberra, 216 pp. 2004.

KUWAYAMA, S. H. Die Psyllid en Japan. I. Transactions of the Sapporo Natural History Society, Japan, v. 2, p. 149-189, 1908.

LOPES, A. S. et al. Diversidade de insetos e aranhas presentes em diferentes fisionomias no Pantanal, na seca e cheia, Corumbá, MS. Mulitemas, Campo Grande, v. 22, p. 127-154, 2017. DOI: $10.20435 /$ multi. v22i51. 1422

MACHADO, L. M. et al. First record of Heteropsylla caldwelli Burckhardt (Hemiptera: Psyllidae) from Brazil and its population dynamics on earpod tree in Rio Grande do Sul. Revista Brasileira de Entomologia, Curitiba, v. 61, p. 290-293, 2017. DOI: http://dx.doi.org/10.1016/j.rbe.2017.07.003

MALENOVSKÝ, I. et al. Descriptions of two new Pseudophacopteron species (Hemiptera: Psylloidea: Phacopteronidae) inducing galls on Aspidosperma (Apocynaceae) in Brazil. Acta Entomologica Musei Nationalis Pragae, Praga, v. 55, p. 513-538, 2015. Available from: http:// zoobank.org/urn:lsid:zoobank.org:pub:1451DAF4-3F24-496C-86B5-3EEF4B9C5261

MATHUR, R. N. Psyllidae of the Indian Subcontinent. New Delhi: Indian Council of Agricultural Research, 1975. 429 p.

MAZZARDO, T. et al. Diversity and distribution of jumping plant-lice (Hemiptera: Psylloidea) along edges of Amazon-Cerrado transitional forests in Sorriso, Mato Grosso, Brazil. CheckList, São Paulo, v. 13, p. 1-7, 2017. DOI: https://doi.org/10.15560/13.3.2131

MAZZARDO, T. et al. Registro de Isogonoceraia divergipennis White; Hodkinson associado a Poincianella pluviosa (Fabaceae) em Mato Grosso, Brasil. Arquivos do Instituto Biológico, Campinas, v. 83, p. 1-3, 2016. DOI: http://dx.doi.org/10.1590/1808-1657000642014.

MOORE, K. M. Observations on some Australian forest insects. 17. Two new species of Glycaspis (Homoptera: Psyllidae) and a note on Glycaspis occidentalis (Solomon). Proceedings of the Linnean Society of New South Wales, Australia, v. 89, p. 148-151, 1964.

NUNES, J. R. S.; SILVA, C. J.; FERRAZ, L. Mato Grosso e seus biomas: Biodiversidade, Desafios Sócio Ambientais, Unidades de Conservação Iniciativas de Políticas Públicas e Privadas para a Conservação. Gestão Universitária, Brazil, v. 7, p. 01-28, 2017. Available from: http://www. gestaouniversitaria.com.br/artigos-cientificos/mato-grosso-e-seus-biomas-biodiversidadedesafios-socio-ambientais-unidades-de-conservacao-iniciativas-de-politicas-publicas-eprivadas-para-a-conservacao. Accessed: 08 jul. 2020.

OUVRARD, D. Psyl'list - The World Psylloidea Database. [S. l.: s. n.], 2019. Available from: http://www.hemiptera-databases.com/psyllist. Accessed: 19 sep. 2019.

PERES FILHO, O. et al. Registro de ocorrência de Glycaspis brimblecombei (Moore, 1964) (Hemiptera: Psyllidae) e seus inimigos naturais em povoamentos de Eucalyptus spp. no estado de Mato Grosso. Revista de Agricultura, São Piracicaba, v. 86, p. 122-124, 2011. Available from: http://www.fealq. 
org.br/ojs/index.php/revistadeagricultura/article/view/2862/pdf_2432

QUEIROZ, D. L.; BURCKHARDT, D.; ANJOS, N. Psilídeos no Brasil: 8 - Mastigimas anjosi (Hemiptera, Psylloidea), nova praga da Toona ciliata no Brasil. Brasília: EMBRAPA, 2013. Available from: https://ainfo.cnptia.embrapa.br/digital/bitstream/item/82815/1/CT-313-Dalvafinal.pdf. Accessed: 15 sep. 2019.

QUEIROZ, D. L. et al. New country, Brazilian state and host records of the eucalypt shoot psyllid, Blastopsylla occidentalis (Hemiptera: Psylloidea). Pesquisa Florestal Brasileira, Brasília, v. 38, p. 1-4, 2018. DOI: 10.4336/2018.pfb.e201701533

SALIBA, I.L. et al. First record of Glycaspis brimblecombei (Moore, 1964) and Blastopsylla occidentalis (Taylor, 1985) (Hemiptera, Aphalaridae) in eucalyptus plantations in State of Pará, Brazil. Entomological Communications, v. 1, p. 1-3, 2019. DOI: https://doi.org/10.37486/26751305.ec01009

SANTANA, D. L. Q. Psilídos no Brasil: 3 - Blastopsylla occidentalis Taylor, 1985 - Hemiptera: Psyllidae. Colombo: EMBRAPA Florestas, 2008. 4 p. (EMBRAPA Florestas. Comunicado técnico, 204). Available from: https://ainfo.cnptia.embrapa.br/digital/bitstream/CNPF-2009-09/44431/1/ com_tec204.pdf. Accessed: 15 sep. 2019.

SANTANA, D. L. Q.; BURCKHARDT, D. Isogonoceraia divergipenis (Hemiptera: Psylloidea) em Sibipiruna no Brasil. In: CONGRESSO BRASILEIRO DE ENTOMOLOGIA, 19., 2002, Manaus. Anais [...], Manaus: INPA, 2002. p.150.

SANTANA, D. L. Q.; BURCKHARDT, D. A new triozid pest (Hemiptera, Psylloidea, Triozidae) on ornamental Trumpet Trees (Tabebuia spp., Bignoniaceae) in Brazil. Revue Suisse de Zoologie, Suisse, v. 108, n. 3, p. 541-550, 2001.

SILVA, A. L. et al. Dinâmica populacional de Glycaspis brimblecombei e inimigos naturais em Eucalyptus spp. Cuiabá-MT. Floresta e Ambiente, Rio de Janeiro, v. 20, p. 80-90, 2013. DOI: http://dx.doi.org/10.4322/floram.2012.066.

SILVA, J. M. C.; BATES, J. Biogeographic patterns and conservation in the south American Cerrado: A tropical Savanna Hotspot, Bioscience, Uberlândia, v. 52, p. 225-234, 2002. DOI: https://doi.org/10.1641/0006-3568(2002)052[0225:BPACIT]2.0.CO;2

TAYLOR, G. S. The taxonomic status of Schedotrioza multitudinea (Maskell) (Psylloidea: Triozidae) with notes on its biology. Journal of the Australian Entomological Society, Australia, v. 24, p. 305-312, 1985.

WHITE, I. M.; HODKINSON, I. D. New psyllids (Homoptera, Psylloidea) from the cocoa region of Bahia, Brazil. Revista Brasileira de Entomologia, Curitiba, v. 24, p. 75-84, 1980. 